\title{
A Scalable Method for Thickness and Lateral Engineering of 2D Materials
}

Sun, Jianbo; Giorgi, Giacomo; Palummo, Maurizia; Sutter, Peter; Passacantando, Maurizio; Camilli, Luca

Published in:

ACS Nano

Link to article, DOI:

10.1021/acsnano.0c00836

Publication date:

2020

Document Version

Publisher's PDF, also known as Version of record

Link back to DTU Orbit

Citation (APA):

Sun, J., Giorgi, G., Palummo, M., Sutter, P., Passacantando, M., \& Camilli, L. (2020). A Scalable Method for Thickness and Lateral Engineering of 2D Materials. ACS Nano, 14(4), 4861-4870.

https://doi.org/10.1021/acsnano.0c00836

\section{General rights}

Copyright and moral rights for the publications made accessible in the public portal are retained by the authors and/or other copyright owners and it is a condition of accessing publications that users recognise and abide by the legal requirements associated with these rights.

- Users may download and print one copy of any publication from the public portal for the purpose of private study or research.

- You may not further distribute the material or use it for any profit-making activity or commercial gain

- You may freely distribute the URL identifying the publication in the public portal 


\title{
A Scalable Method for Thickness and Lateral Engineering of 2D Materials
}

\author{
Jianbo Sun, Giacomo Giorgi, Maurizia Palummo, Peter Sutter, Maurizio Passacantando, \\ and Luca Camilli*
}

Cite This: https://dx.doi.org/10.1021/acsnano.0c00836

Read Online

ABSTRACT: The physical properties of two-dimensional (2D) materials depend strongly on the number of layers. Hence, methods for controlling their thickness with atomic layer precision are highly desirable, yet still too rare, and demonstrated for only a limited number of 2D materials. Here, we present a simple and scalable method for the continuous layer-by-layer thinning that works for a large class of $2 \mathrm{D}$ materials, notably layered germanium

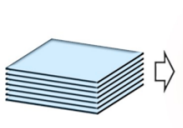

Original Flake
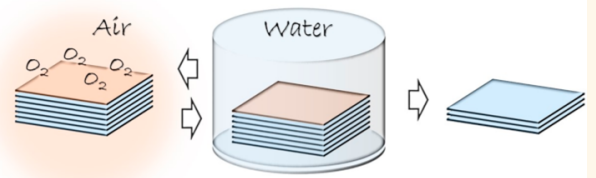

oxidation/Etching cycles pnictides and chalcogenides. It is based on a simple oxidation/ etching process, which selectively occurs on the topmost layers. Through a combination of atomic force microscopy, $\mathrm{X}$-ray photoelectron spectroscopy, Raman spectroscopy, and X-ray diffraction experiments we demonstrate the thinning method on germanium arsenide ( $\mathrm{GeAs})$, germanium sulfide ( $\mathrm{GeS})$, and germanium disulfide $\left(\mathrm{GeS}_{2}\right)$. We use first-principles simulation to provide insights into the oxidation mechanism. Our strategy, which could be applied to other classes of $2 \mathrm{D}$ materials upon proper choice of the oxidation/etching reagent, supports 2D material-based device applications, e.g., in electronics or optoelectronics, where a precise control over the number of layers (hence over the material's physical properties) is needed. Finally, we also show that when used in combination with lithography, our method can be used to make precise patterns in the 2D materials.

KEYWORDS: 2D materials, oxidation, layer-by-layer thinning, GeAs, GeS, GeS

I nspired by the successful isolation of graphene in $2004,{ }^{1}$ layered two-dimensional (2D) materials with their various fascinating properties have become one of the most popular research topics in materials science. ${ }^{2}$ Although significant progress has been made during the past 16 years, the integration of these materials into functional devices is often limited by the lack of control over their thickness, i.e., the number of individual layers, which in turn has a crucial influence on the materials' physical properties. ${ }^{3-7}$ Methods for controlling the thickness of $2 \mathrm{D}$ materials with atomic layer precision are therefore highly desirable.

These methods can be divided into bottom-up processes, where the thickness of a $2 \mathrm{D}$ material is controlled during its synthesis, and top-down approaches, where the thickness is instead controlled (i.e., reduced down to the chosen value) $a$ posteriori. The latter strategy has the advantage that it could more easily produce junctions, patterns, or superlattices with a designed layer number (e.g., monolayer-bilayer junctions) when applied locally on selected areas. Thus far, only a few top-down methods have been reported, and they involve physical processes, such as plasma, ${ }^{8,9}$ focused ion beam, ${ }^{10}$ or laser etching. ${ }^{11}$ These processes require costly equipment and are suitable to work only over limited areas. Furthermore, they can damage the $2 \mathrm{D}$ material by introducing defects such as vacancies, heteroatoms within the crystal lattice, or functional groups on the surface. Moreover, their efficacy has only been demonstrated so far for a limited number of $2 \mathrm{D}$ materials, namely $\mathrm{MoS}_{2}$ or black phosphorus (BP). Therefore, it would be beneficial to find an alternative, scalable strategy, which could be applied to most $2 \mathrm{D}$ materials without inducing structural damage.

In this paper, we present a simple chemical approach that can be used to reduce the thickness of $2 \mathrm{D}$ materials with (sub)monolayer precision and is based on a scalable and controllable oxidation/etching process. The top layer(s) of a given 2D material-or part thereof-is first oxidized in air; the oxidized regions are then selectively removed upon immersion in a proper etchant. In a way, this process is very similar to what happens when a native oxide is etched away from the surface of conventional semiconductors, such as $\mathrm{Ge}$ and $\mathrm{Si}$,

Received: January 30, 2020

Accepted: March 10, 2020

Published: March 10, 2020 
upon immersion in water or hydrochloric acid for $\mathrm{Ge}$ and hydrofluoric acid for Si. Here, we demonstrate that our thinning strategy works for several germanium-based 2D materials, namely, GeAs, GeS and $\mathrm{GeS}_{2}$. Previous literature indicates that the same strategy could also be extended to other $2 \mathrm{D}$ materials. ${ }^{12,13}$

\section{RESULTS AND DISCUSSION}

GeAs is a layered material that has recently attracted intensive interest due to a bandgap in a technologically relevant energy region $(0.5-2 \mathrm{eV} \text {, depending on the number of layers })^{14,15}$ and strongly anisotropic electrical and optical properties. ${ }^{16,17}$ As for the case of other $2 \mathrm{D}$ materials, for instance, $\mathrm{BP}^{18}$ and silicene, ${ }^{19}$ previous reports lead us to believe that GeAs suffers from degradation when exposed to ambient air. ${ }^{17} \mathrm{X}$-ray photoelectron spectroscopy (XPS) performed on GeAs flakes exfoliated on an $\mathrm{Au} / \mathrm{SiO}_{2} / \mathrm{Si}$ substrate and then left in air for a few days indeed confirms changes to the material (Figure 1). After air exposure, the $\mathrm{Ge} 3 \mathrm{~d}$ core level features two contributions, one at $29.7 \mathrm{eV}$ and one at around $32.8 \mathrm{eV}$ (Figure 1b). The former is very close in energy to the peak found for $\mathrm{GeAs}_{2}$ at $29.5 \mathrm{eV}^{20}$ and can therefore be attributed to

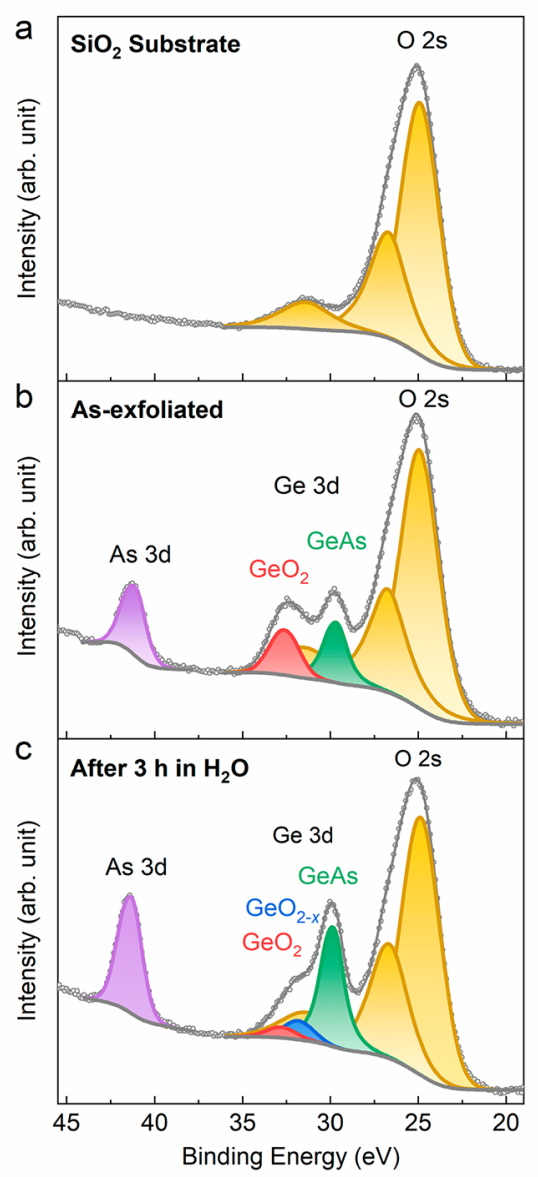

Figure 1. Chemical evolution of GeAs flakes upon exposure to air and subsequent immersion in water. (a) XPS spectrum collected in an area of the substrate without GeAs flakes. (b) XPS spectrum of GeAs flakes after being exposed to air at ambient conditions for a few days. (c) XPS spectrum of GeAs flakes after $3 \mathrm{~h}$ immersion in water. All spectra are normalized with respect to the intensity of $\mathrm{C}$ 1 s peak. the Ge-As bond. The latter is significantly shifted toward higher binding energies and is due to the formation of germanium oxide $\left(\mathrm{GeO}_{2}\right){ }^{21-23}$ The ratio between these two peaks is 1.2, proving the high degree of oxidation of the material within the probed volume. Interestingly, the As 3d core level shows only one peak at around $41 \mathrm{eV}$, while other possible features at higher binding energies due to arsenic oxide are absent. ${ }^{24}$ Considering their sensitivity factors, the concentration of Ge $3 \mathrm{~d}$ and As $3 \mathrm{~d}$ due to GeAs bond is $51 \%$ and $49 \%$, respectively. These findings suggest that the surface of GeAs after exposure to air is partially oxidized, with the possible oxidation mechanism involving the loss of As and the formation of germanium oxide. The oxidation mechanism will be discussed in more detail later on in the text.

Since oxidation is often seen as being detrimental to the properties of materials and devices (e.g., it could introduce scattering sources that reduce the carrier mobility), the exfoliated GeAs flakes were immersed in deionized (DI) water to remove the "native" oxide. This is a procedure commonly used for Ge structures ${ }^{25-27}$ and takes advantage of the water solubility of $\mathrm{GeO}_{2} \cdot{ }^{28}$ As shown in Figure $1 \mathrm{c}$, after immersion in deionized water for $3 \mathrm{~h}$, the Ge $3 \mathrm{~d}$ peak corresponding to $\mathrm{GeO}_{2}$ is significantly reduced to about $10 \%$ of the intensity of $\mathrm{Ge}$ coordinated to As. In addition, a new signal is found at $31.8 \mathrm{eV}$ stemming from the presence of $\mathrm{Ge}$ suboxides $\left.\left(\mathrm{GeO}_{2-\mathrm{x}}\right)\right)^{21,24}$ which is probably due to an incomplete removal of $\mathrm{GeO}_{2}$ by water. Furthermore, the intensity ratio between the $\mathrm{Ge} 3 \mathrm{~d}$ component due to $\mathrm{Ge}-\mathrm{As}$ bond and the As $3 \mathrm{~d}$ core level is unchanged. Taken together, these results indicate that water can effectively remove the oxidized areas on top of the GeAs flakes.

In order to probe how the oxidation/etching process could affect the morphology of GeAs, the process was repeated four times for a given flake while at the end of every etching step atomic force microscopy (AFM) was used for inspection. In the experiment reported in Figure 2, the oxidation step consists of $4 \mathrm{~h}$ exposure in air at ambient conditions, whereas the etching is carried out through $20 \mathrm{~h}$ immersion in $\mathrm{O}_{2}$-depleted water. More experimental details are given in the Methods. Figure 2a shows the AFM images taken immediately after exfoliation, and then again after each etching step. First, it can be noticed that the lateral morphology of the flake remains unchanged throughout the whole experiment and no features due to degradation, for instance, pits or blisters, can be observed on the surface. Instead, a gradual and continuous thinning of the flake is observed. To quantitatively evaluate the thinning, cross section profiles are taken along the dashed lines in the AFM images in Figure 2a. From the profiles reported in Figure $2 b$, two pieces of information can be inferred: first, the reduction in thickness after each oxidation/etching cycle appears to be constant, and second, the roughness of the flake appears to be unchanged as well. To visualize the reduction in thickness for the entire flake (and not only along one line), we report in Figure $2 c$ the height distributions extracted from the AFM images in Figure 2a, with the peaks for the $\mathrm{SiO}_{2}$ substrate being centered at zero. By fitting the height distribution with Gaussian peaks, we can evaluate the thickness of the flake. The evolution of the mean thickness determined from these Gaussians is shown in Figure 2d. It is clear that the thickness of the whole flake is indeed reduced by nearly the same amount after each oxidation/etching cycle, proving that the process is quite reliable, repeatable, and independent of the previous etching history of the flake. The average thickness 

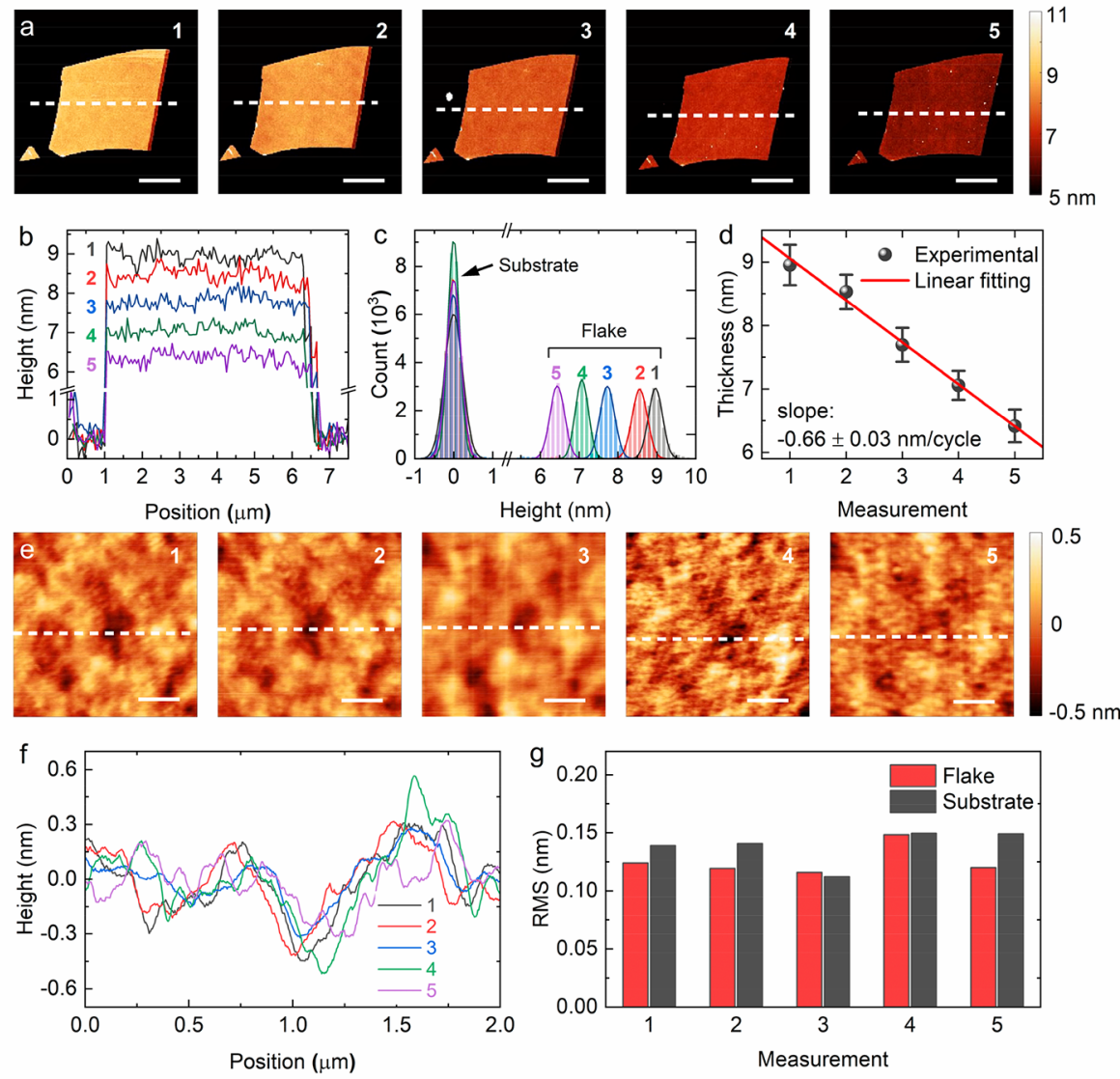

Figure 2. Morphological characterization of the GeAs flakes upon oxidation/etching thinning. (a) AFM images of the same flake taken (1) after exfoliation, (2) after the first, (3) the second, (4) the third and (5) the fourth oxidation/etching cycle (scale bars: $2.5 \mu \mathrm{m}) .(\mathrm{b})$ Cross section profiles along the dashed lines in the AFM images in (a). (c) Height distribution of the AFM images in (a), fitted with Gaussian peaks. (d) Thickness of the flake as extracted from the Gaussian fitting. (e) High-resolution AFM images of the same region on the flake surface taken after (1) exfoliation, (2) the first, (3) the second, (4) the third, and (5) the fourth oxidation/etching cycle (scale bars: $0.5 \mu \mathrm{m}$ ). (f) Cross section profiles collected along the dashed lines in the AFM images in (e). (g) RMS roughness of the GeAs flake and substrate after exfoliation and after each oxidation/etching step.
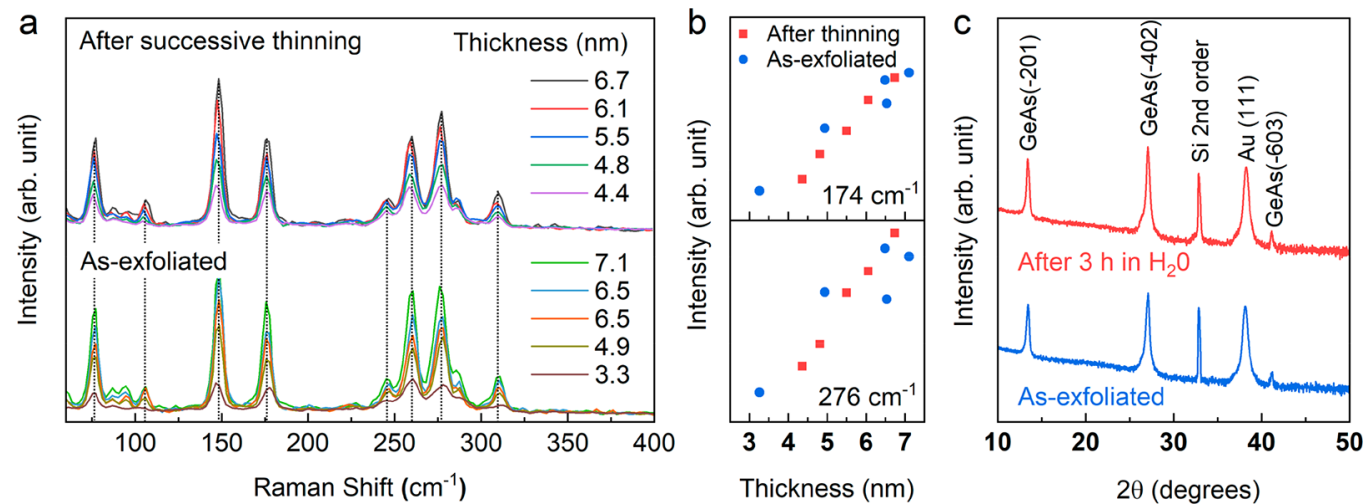

Figure 3. Structural investigation of GeAs flakes upon oxidation/etching thinning. (a) Raman spectra of a GeAs flake after successive oxidation/etching cycles (upper curves) and of as-exfoliated GeAs flakes with different thickness (bottom curves). (b) Intensity of the Raman peaks at 174 and $276 \mathrm{~cm}^{-1}$ in (a) as a function of the flake thickness for both thinned (red squares) and as-exfoliated (blue dots) samples. (c) XRD spectrum of GeAs flakes following exfoliation onto a $\mathrm{SiO}_{2} / \mathrm{Si}$ substrate (top curve) and then again after one oxidation/ etching treatment (bottom curve). The Au stems from a $2 \mathrm{~nm}$ thick $\mathrm{Au}$ film on the $\mathrm{SiO}_{2} / \mathrm{Si}$ substrate.

reduction per cycle amounts to $0.66 \mathrm{~nm}$, which coincides with the interlayer distance in GeAs. ${ }^{16,17}$ This shows that, under the conditions used in this experiment, the oxidation/etching method produces a layer-by-layer thinning of the GeAs flake. Moreover, considering that the root-of-mean-square (RMS) roughness is mathematically equivalent to the standard deviation of the Gaussian distribution, the nearly constant width of the Gaussian peaks for the flake in Figure 2c implies that the surface roughness is not affected by the oxidation/ etching cycle. To further evaluate the surface roughness, we 

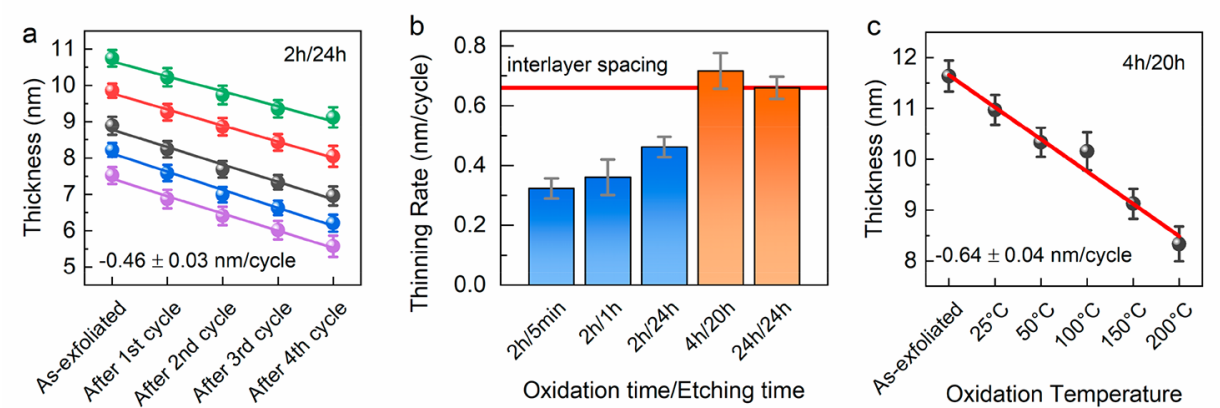

Figure 4. Effects of oxidation time, etching time and oxidation temperature. (a) Thickness evolution of five GeAs flakes measured after (1) exfoliation, (2) the first, (3) the second, (4) the third, and (5) the fourth cycle. The oxidation/etching times are $2 \mathrm{~h} / 24 \mathrm{~h}$, and the average of the five slopes is taken as the thinning rate for the corresponding oxidation/etching time. (b) Thinning rates with different oxidation/ etching time combinations; the horizontal red line indicates the interlayer distance of GeAs. (c) Thickness evolution of a GeAs flake upon oxidation/etching processing with increasing oxidation temperatures.

performed additional AFM measurements with higher resolution. Figure 2e displays AFM images taken on the same region of the GeAs flake after successive oxidation/ etching cycles. The main features of the flake after exfoliation remain visible throughout the experiment. This is also highlighted by the cross section profiles taken across such features (displayed in Figure 2f), which show that the surface fluctuations (peak-to-valley distance in the height profiles) are also unchanged. These results indicate that the oxidation/ etching cycles do not degrade the flake surface, but reduce homogeneously the flake thickness with atomic layer precision (i.e., layer-by-layer). The fluctuations observed on the flake surface are likely due to the roughness of the underlying $\mathrm{SiO}_{2}$ substrate, as (i) such features are also observed on the bare substrate (see Supporting Information Figure S2) and (ii) they exist already on the as-exfoliated flake and remain unchanged throughout the experiment. In Figure 2g, the RMS roughness of the whole GeAs flake surface is plotted in comparison with that of the substrate. No increase of the roughness is detectable after the oxidation/etching processes and the roughness of the flake remains low (less than $0.15 \mathrm{~nm}$ ), comparable to that of the substrate.

Raman spectroscopy and X-ray diffraction (XRD) were employed to examine the structural integrity of the GeAs flakes after the oxidation/etching process. The Raman spectra of an individual flake after successive oxidation/etching cycles are reported in Figure 3a (upper curves) after normalization with respect to the $\mathrm{Si}$ peak at $521 \mathrm{~cm}^{-1}$. As a comparison, we also show in the same figure the Raman spectra of as-exfoliated GeAs flakes with similar thickness (bottom curves). Both sets of spectra show the characteristic modes of GeAs. ${ }^{16,17}$ Moreover, it is evident that regardless of the way the thinning is carried out (i.e., exfoliation or oxidation/etching) the intensity of the peaks decreases approximately linearly as the thickness of the flake decreases. Figure $3 \mathrm{~b}$ shows that the intensity of the two representative peaks at 147 and $276 \mathrm{~cm}^{-1}$ in both sets of spectra exhibits similar thickness-dependence. The XRD measurements (Figure 3c) performed on a sample with a large number of $\mathrm{GeAs}$ flakes deposited on a $\mathrm{Au} / \mathrm{SiO}_{2} / \mathrm{Si}$ substrate also confirms that the crystal structure of $\mathrm{GeAs}^{15}$ remains unchanged after the oxidation/etching treatment. Furthermore, we can use the XRD spectrum to confirm the interlayer spacing, which is $0.66 \mathrm{~nm}$, consistent with previous reports. $^{15,17}$
To gain further insights into the thinning mechanism, we carried out a more detailed study by systematically varying the key parameters in this process, namely the oxidation time, the etching time and the oxidation temperature. Five sets of experiments were conducted with different oxidation/etching time combinations and for each set of experiment, five flakes with different initial thicknesses were examined. For these five sets of experiments, the samples were oxidized in air at room temperature. Similar to the previous experiment, we used AFM to follow the thickness evolution of the flakes. The results of the experiment with $2 \mathrm{~h} / 24 \mathrm{~h}$ oxidation/etching time are plotted in Figure $4 \mathrm{a}$ as an example, while those from the other sets of experiments are given in Supporting Information Figure S3. First, in each experiment all five flakes show a gradual thinning after every oxidation/etching cycle, just as observed in Figure 2. Moreover, the reduction in thickness is comparable for all the five flakes in each experiment. To allow for a comparative analysis, we calculated the average thickness reduction per oxidation/etching cycle of the five flakes in each experiment, which can be considered as the thinning rate. The results are summarized in Figure $4 \mathrm{~b}$. First, we focus on the experiments performed with the shortest oxidation time, that is $2 \mathrm{~h}$. For an immersion time in water of $5 \mathrm{~min}$, the thinning rate is $0.32 \pm 0.03 \mathrm{~nm} /$ cycle; the same value is found if the immersion time is brought to $1 \mathrm{~h}(0.36 \pm 0.06 \mathrm{~nm} /$ cycle $)$, whereas it increases only slightly to $0.43 \pm 0.03 \mathrm{~nm} /$ cycle when the immersion time is as long as $24 \mathrm{~h}$. Clearly, the thickness reduction is limited by the short oxidation time used in this set of experiments and, interestingly, it is still smaller than the interlayer distance in GeAs. Thus, less than one full GeAs layer is removed at each cycle. Here, it is worth noting that also in this case, i.e., in the case of incomplete oxidation of the topmost layer, the surface roughness of the GeAs flakes is still constant throughout the experiment (Supporting Information Figures S4 and S5). In other words, we did not detect any area of changing thickness (holes or islands). This implies that the thinning occurs homogeneously over the entire surface and not in a "reversed island growth" mode. Consequently, the etched areas (i.e., the areas of the top layer removed upon immersion in water) are small, and in this case smaller than $10 \mathrm{~nm}$, which is the lateral resolution of our AFM setup as given by the radius of the probe. On the other hand, if the oxidation time is increased to $4 \mathrm{~h}$, with each etching step the thickness of the flake is reduced by exactly one layer (the horizontal red line in Figure $4 \mathrm{~b}$ represents the 

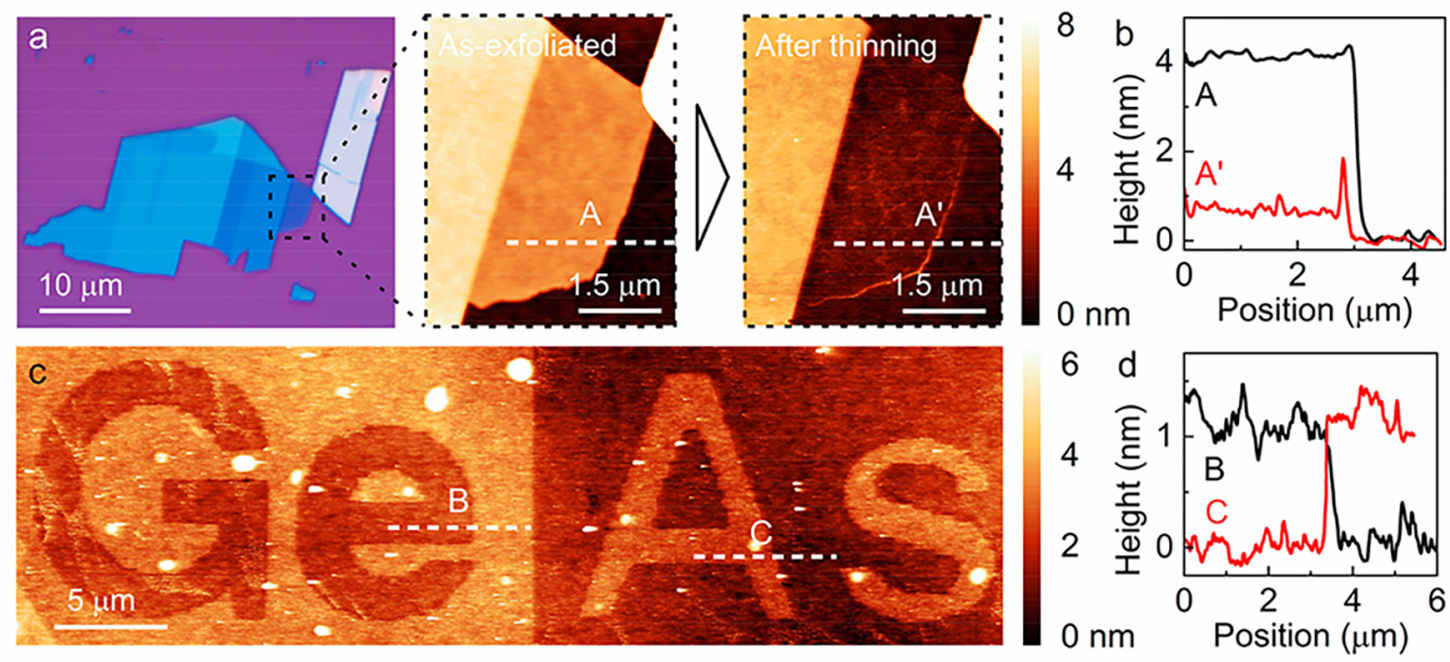

Figure 5. Approaching the single layer limit and lateral patterning of the GeAs surface. (a) From left to right: optical image of an asexfoliated GeAs flake; AFM image of the selected area obtained after exfoliation; AFM image taken in the same area after five oxidation/ etching cycles. (b) Cross section profiles along the dashed lines labeled as A and A' in (a). (c) AFM image of the "GeAs" pattern on a GeAs flake. (d) Cross section profiles along the dashed lines labeled as B and $\mathrm{C}$ in (c).
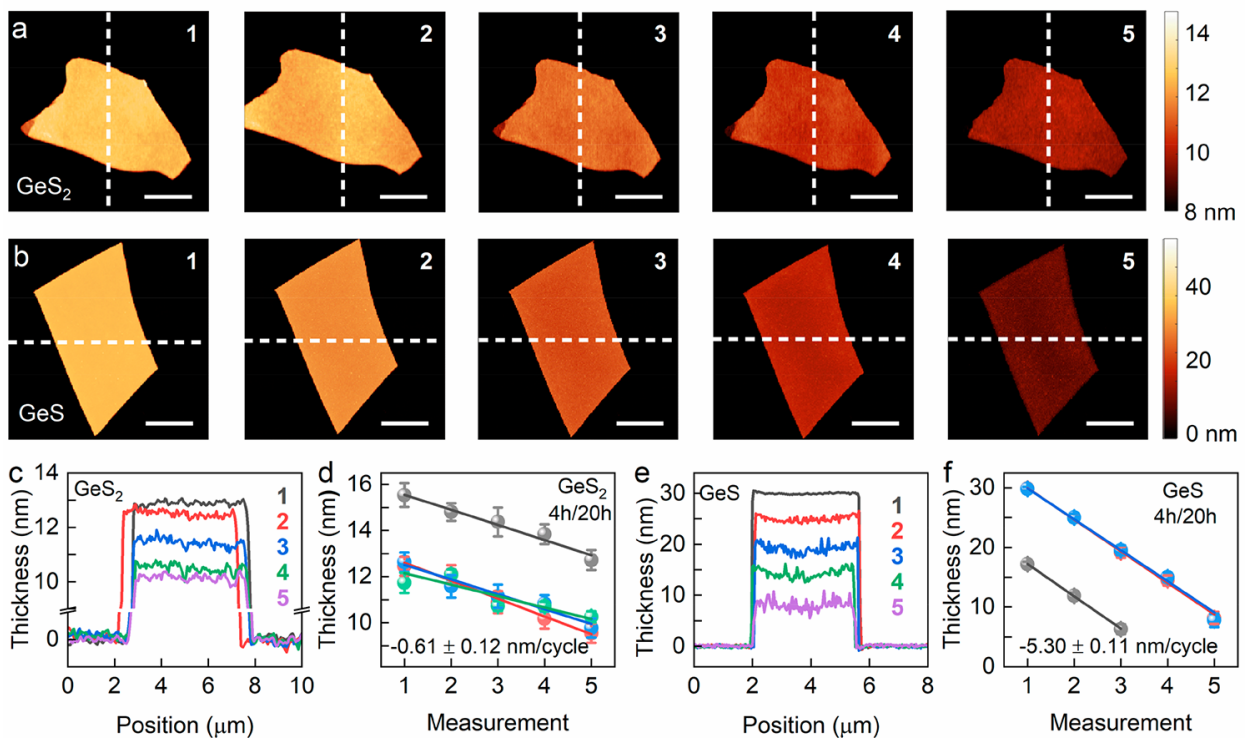

Figure 6. Thinning of layered $\mathrm{GeS}_{2}$ and GeS. AFM images of the (a) $\mathrm{GeS}_{2}$ and (b) GeS flakes that were taken after (1) exfoliation, (2) the first, (3) the second, (4) the third, and (5) the fourth oxidation/etching $(4 \mathrm{~h} / 20 \mathrm{~h})$ cycle. The scale bar is $2 \mu \mathrm{m}$ in (a) and $2.5 \mu \mathrm{m}$ in (b). (c) Cross section profiles along the dashed lines in (a). (d) Thickness evolution of the $\mathrm{GeS}_{2}$ flake shown in (a). (e) Cross section profiles along the dashed lines in (b). (f) Thickness evolution of the GeS flake shown in (b).

measured interlayer distance in GeAs, namely $0.66 \mathrm{~nm})$. Since the same thickness reduction is achieved when the oxidation time is further increased to $24 \mathrm{~h}$, we can conclude that the oxidation of GeAs is a self-limiting process, in the sense that once the topmost layer becomes fully oxidized, further oxidation of the layers underneath is prevented, or at least significantly slowed down. The top oxide layer acts as a passivating film, protecting the "bulk", as it happens for the native oxides in the cases of $\mathrm{Ge}$ or $\mathrm{Si}$, for instance. We further explored if this self-limiting nature of the oxidation/thinning process still remains at higher oxidation temperatures. In Figure $4 c$, the samples were oxidized at increasing temperatures (from room temperature to $200{ }^{\circ} \mathrm{C}$ ) for $4 \mathrm{~h}$, followed by $20 \mathrm{~h}$ etching in oxygen-depleted water. The thickness reduction per cycle still amounts to one layer, regardless of the oxidation temperature that was used.

The data reported so far demonstrate that the thickness of GeAs can be controlled with (sub-) monolayer precision by a simple process involving oxidation in air and subsequent immersion in oxygen-depleted water. To reach a certain final thickness, one can adjust the number of oxidation/etching cycles to reduce the thickness by a desired number of layers. As shown in Figure 5a, for instance, after five successive oxidation/etching cycles, the GeAs flake under study was thinned from $4.2 \mathrm{~nm}$ down to $0.7 \mathrm{~nm}$, thus achieving singlelayer thickness. Here, it is worth noting that to the best of our knowledge no group has so far been able to obtain GeAs flakes with single-layer thickness. Thus, our method can indeed be 
used to provide single-layer flakes for a number of those 2D layered materials that are otherwise extremely challenging to obtain via, for instance, standard exfoliation technique.

Furthermore, this method when coupled with lithographic patterning could even be used to define patterns of different thicknesses on the $2 \mathrm{D}$ material's surface. In Figure 5c, we demonstrate such a pattern with depth of around $1.1 \mathrm{~nm}$ that was fabricated on a GeAs flake using the oxidation/etching method in combination with electron-beam lithography (EBL).

Now, it would be remarkable if the same process could be successfully applied to other layered materials. In this context, since the crucial (and limiting) step in the proposed approach is the formation of "native" germanium oxide, we have performed experiments similar to the ones described above on other Ge-based layered materials, namely on $\mathrm{GeS}$ and $\mathrm{GeS}_{2}{ }^{20,29,30}$ Here, it is worth mentioning that GeAs, GeS, and $\mathrm{GeS}_{2}$ are three very different compounds with different crystal structures. The only similarity is the fact that they all contain $\mathrm{Ge}$, although with different oxidation states. As shown in Figure 6, also for the case of $\mathrm{GeS}_{2}$ and $\mathrm{GeS}$ the thickness of the flakes constantly decreases after every oxidation/etching cycle, while no significant change of the lateral morphology can be observed. The integrity of the flakes after the oxidation/ etching process is confirmed by Raman spectroscopy (see Supporting Information Figure S6). Interestingly, there is a remarkable difference between $\mathrm{GeS}_{2}$ and $\mathrm{GeS}$ that deserves further attention. Namely, while in the former case the flakes show a thinning rate of around $0.61 \pm 0.12 \mathrm{~nm} /$ cycle, which implied the removal of one layer $(0.57 \mathrm{~nm}$ being the interlayer distance in $\left.\mathrm{GeS}_{2}\right)$, ${ }^{31}$ in the latter the thinning rate is as high as $5.30 \mathrm{~nm} /$ cycle, even though the oxidation and etching time for $\mathrm{GeS}$ and $\mathrm{GeS}_{2}$ were the same. Moreover, the roughness of $\mathrm{GeS}$ surface increases during the oxidation/etching process, as shown by the line profiles in Figure 6e. Apparently, within our experimental conditions, $\mathrm{GeS}$ oxidizes at a much higher rate than $\mathrm{GeAs}$ and $\mathrm{GeS}_{2}$, and while the oxidation of GeAs and $\mathrm{GeS}_{2}$ is a self-limiting process, the same does not apply to $\mathrm{GeS}^{32}$ Supporting Information Figure S7 shows that the thinning rate of $\mathrm{GeS}$ can be reduced by using shorter oxidation times, confirming the degree of tunability of the approach reported here.

To identify the possible reasons behind the different behavior observed in $\mathrm{GeS}$ and $\mathrm{GeS}_{2}$, we have performed $a b$ initio calculations on their quadrilayer (4L) structures (see Supporting Information Figure N1). Such structures are more similar than monolayers to the relatively thick flakes that we have used as starting points in our experiments. Within our framework, we have expanded previous theoretical studies performed on binary group-IV monochalcogenide monolayers (among which $\mathrm{GeS})^{33}$ showing that substitutional oxygen $\left(\mathrm{O}_{\mathrm{S}}\right)$ forms spontaneously at the chalcogen sites in the presence of chalcogen vacancies $\left(\mathrm{V}_{\mathrm{S}}\right)$ and oxygen. Experimentally, the assumption that this is the preferential pathway for oxidation is also supported by the fact that the same occurs in other chalcogen-containing 2D materials, for instance, transition metal dichalcogenides (TMDs). ${ }^{13,34-37}$ (Moreover, we observe the same to occur in GeAs, as we do not see any sign of As oxides in XPS; see Figure 1.) Finally, to account for possible cooperative effects, we compare systems in which there are two substitutional oxygens rather than only one. The results are summarized in Table 1 (details of the calculations can be found in Supporting Information Note 1).
Table 1. Calculated formation energy $\left(E_{f}\right)$ of two $V_{S}$ and two $\mathrm{O}_{\mathrm{S}}$ in $4 \mathrm{~L}$ of $\mathrm{GeS}$ and $4 \mathrm{~L}$ of $\mathrm{GeS}_{2}{ }^{a}$

$\begin{array}{lcccc} & \mathrm{V}_{\mathrm{S}}{ }^{1-1}(\mathrm{eV}) & \mathrm{V}_{\mathrm{S}}{ }^{1-2}(\mathrm{eV}) & \mathrm{O}_{\mathrm{S}}{ }^{1-1}(\mathrm{eV}) & \mathrm{O}_{\mathrm{S}}{ }^{1-2}(\mathrm{eV}) \\ \mathrm{GeS} & 0.98 & 1.13 & -2.86 & -2.85 \\ \mathrm{GeS}_{2} & 1.19 & 0.86 & -2.52 & -2.85\end{array}$

${ }^{a} \mathrm{~V}_{\mathrm{S}}$ and $\mathrm{O}_{\mathrm{S}}$ both in the uppermost layer $\left(\mathrm{V}_{\mathrm{S}}{ }^{1-1}\right.$ and $\mathrm{O}_{\mathrm{S}}{ }^{1-1}$, respectively) or one in the uppermost and the other in the second uppermost layer $\left(\mathrm{V}_{\mathrm{S}}{ }^{1-2}\right.$ and $\mathrm{O}_{\mathrm{S}}{ }^{1-2}$, respectively) are considered.

The first consideration is that although the vacancy formation is always an endothermic process (formation energy, $E_{\mathrm{f}}>0$ ), the second $\mathrm{V}_{\mathrm{S}}$ thermodynamically costs almost the same in both $4 \mathrm{~L}$ systems, with the second vacancy in the uppermost layer $\left(\mathrm{V}_{\mathrm{S}}{ }^{1-1}\right)$ being more easily formed in the case of $\mathrm{GeS}(0.98 \mathrm{eV})$ than in $\mathrm{GeS}_{2}(1.19 \mathrm{eV})$. Second, in regard to the formation of the second $\mathrm{O}_{\mathrm{S}}$, we notice that this is always a highly exothermic process $\left(E_{\mathrm{f}}<0\right)$.

On the basis of these $E_{\mathrm{f}}$ values, we can conclude that thermodynamically the introduction of multiple substitutional oxygens in $\mathrm{GeS}$ is equally favored to occur both in the external and internal layer, which confirms the strong tendency of $\mathrm{GeS}$ (bulk) toward oxidation. In the case of $\mathrm{GeS}_{2}$, instead, the $E_{\mathrm{f}}$ of the second oxygen defect in the first layer is less favored (i.e., lower affinity toward oxidation), which may help explain the experimental observation of a slower oxidation process. Nevertheless, it is important to point out that these theoretical results, although already useful to interpret the experimental observation, describe only the very initial stage(s) of the oxidation process, and cannot therefore be considered as exhaustive. For instance, while so far we have considered only the substitution of two oxygen atoms, oxidation might in fact proceed through the formation of substoichiometric oxides ${ }^{38-42}$ and the self-limiting process observed for $\mathrm{GeS}_{2}$ and GeAs but not for GeS could be related to a larger strain occurring in the former cases with respect to the latter (see Supporting Information Note 2). The presence of a largely strained interface has been indeed invoked to explain the selflimiting oxidation of silicon nanostructures. ${ }^{43-46}$ At this regard, we suggest that $a b$ initio molecular dynamics simulations, which will be the focus of future investigation, could provide more satisfactory answers on the presence of substoichiometric oxides as well as on the role of kinetic and thermal effects.

\section{CONCLUSION}

To conclude, we have identified a method to reduce the thickness of layered materials with (sub)monolayer precision. This method, based on the formation of a surface oxide layer and its selective removal by a suitable etchant, is highly controlled, scalable, and does not require the use of costly and specialized instrumentation. The efficacy of this approach has been demonstrated for a number of germanium-based layered materials, namely GeAs, GeS, and $\mathrm{GeS}_{2}$. For most of the considered layered crystals (with exception of $\mathrm{GeS}$ ), the oxidation is self-limiting to the outermost layer, which accounts for the ability to controllably reduce the thickness layer by layer. Beyond materials with $\mathrm{Ge}$ as a cation, it is reasonable to suppose that the same strategy can be applied to other layered materials. In this regard, it is important to discuss the case of BP. As shown elsewhere, BP is highly sensitive to oxygen, and air exposure leads to oxidation of $\mathrm{P}$ to $\mathrm{P}_{x} \mathrm{O}_{y}{ }^{12,47-49}$ Similar to $\mathrm{GeO}_{2}$, phosphorus oxides are also soluble in water; ${ }^{12,50}$ this indicates that our strategy demonstrated here for several Ge- 
based layered materials can be applied to BP as well. A similar discussion may be made about other oxygen-sensitive layered materials, whose cation species form surface oxides, for instance $\mathrm{GaSe}{ }^{51}$ given that a proper etchant can be found.

In addition, we consider the case of TMDs. Although in most cases they are more stable in air than the layered materials mentioned earlier, a few studies have shown that it is possible to grow a homogeneous oxide film on top of them via, for instance, thermal oxidation, ${ }^{52}$ exposure to ozone, ${ }^{53}$ or to a focused laser. ${ }^{54}$ Also in this case, the oxidation mechanism involves the loss of the chalcogen atoms, and the formation of transition metal oxides, $\mathrm{MO}_{x}$, with $\mathrm{M}$ being the metal ion of the TMDs under consideration. In fact, since chalcogen vacancies are very common in TMDs, oxidation seems to occur more often than previously expected, ${ }^{34}$ given that oxygen replacing a chalcogenide vacancy is in most cases an exothermic process. ${ }^{35-37}$ Therefore, it is plausible that if a selective etchant for $\mathrm{MO}_{x}$ can be found, our strategy could be applied to TMDs as well. We note here that $\mathrm{W}$ and Mo suboxides, for instance, can indeed be etched by water. ${ }^{13,55}$

Ultimately, in comparison to the methods reported thus far for controlled thinning of $2 \mathrm{D}$ materials, such as $\mathrm{Ar}^{+}$plasma ${ }^{8}$ and laser pruning, ${ }^{56}$ the strategy proposed here is scalable, selflimiting and highly tunable. As controlling the thickness of 2D materials means controlling their electrical, optical and electronic properties, our findings can provide an important contribution to promoting the integration of $2 \mathrm{D}$ materials in a variety of technological fields.

\section{METHODS}

Sample Preparation. GeAs, GeS, and $\mathrm{GeS}_{2}$ (2D Semiconductors Inc.) flakes were exfoliated using thermal release tape (EPL BT-150E$\mathrm{KL}$ ) onto $\mathrm{Si}$ wafer chips with $300 \mathrm{~nm}$ oxide layer. After exfoliating the crystals using the tape for multiple times, the flakes-loaded tape was applied onto the Si chip, which was then transferred onto a hot plate. The tape was released from the Si chip after heating at $75{ }^{\circ} \mathrm{C}$ for 5 $\mathrm{min}$. The samples were used for experiments without further cleaning. To avoid charging issues while performing the XPS experiments, some $\mathrm{SiO}_{2} / \mathrm{Si}$ substrates were coated with an $\mathrm{Au}$ film with nominal thickness of $2 \mathrm{~nm}$ prior to depositing the flakes via exfoliation. Flakes with appropriate size and thickness for AFM characterization were identified using an optical microscope (Nikon L200ND).

Oxidation/Etching Process. The oxidation process was performed in air at room temperature unless otherwise specified. The oxidized layers were removed by immersing the samples in DI water with $\mathrm{N}_{2}$ bubbling $\left(\mathrm{N}_{2}\right.$ purity: $\left.99.999 \%\right)$. Here, we emphasize the necessity of using $\mathrm{O}_{2}$-depleted water (corrosion of the GeAs surface was observed if using DI water without $\mathrm{N}_{2}$ bubbling, as shown in Supporting Information Figure S8). To obtain properly deoxygenated water, the $\mathrm{N}_{2}$ bubbling process was started at least 1 $\mathrm{h}$ before immersing the samples. For the experiments with increasing oxidation temperatures, the samples were heated on a hot plate in air.

Lateral Engineering of GeAs. The lateral engineering of GeAs was realized using our oxidation/etching method in combination with standard EBL process. First, the Si chips with on top the exfoliated GeAs flakes were spin-coated with poly(methyl methacrylate) (PMMA, dissolved in anisole, 4\%). After EBL exposure (LEO SEM 1550 integrated with Raith e-beam writing system) and developing with MIBK/IPA (1:3), the samples were left in air for $12 \mathrm{~h}$ and then immersed in $\mathrm{O}_{2}$-depleted DI water for $12 \mathrm{~h}$. In this way the regions exposed to air and water were thinned down, while those covered by PMMA were not, forming the lateral patterns. The oxidation/etching process was repeated for several times so that the thickness was reduced by a considerable amount. In the end, the PMMA layer was removed with acetone.
Sample Characterization. AFM measurements were performed with a NT-MDT scanning probe microscope in tapping mode using silicon tips (HA-HR series from NT-MDT). The Raman spectra were collected with a DXR xi Raman microscope with a $532 \mathrm{~nm}$ laser $(0.1$ $\mathrm{mW}$ ) and $100 \times$ objectives. XPS experiments were carried out in an ultrahigh vacuum chamber (base pressure $<5 \times 10^{-10}$ Torr) in a PHI 1257 system equipped with a hemispherical analyzer using a nonmonochromatic Al $K \alpha$ X-ray source $(h \nu=1486.6 \mathrm{eV})$. The measurements were performed on the samples right after the introduction into the vacuum chamber, without any annealing. All reported binding energy $(\mathrm{BE})$ data have been calibrated using the $\mathrm{Au}$ $4 \mathrm{f}_{7 / 2}$ peak, centered at a BE of $84.0 \mathrm{eV}$. The intensity of the peaks has been normalized with respect to the intensity of the $\mathrm{C} 1 \mathrm{~s}$ signal of adventitious carbon. All core levels have been fitted with Gaussian functions, following removal of a Shirley background. The XRD characterization was performed using a Bruker D5000 diffractometer with $\mathrm{Cu} K \alpha(\lambda=1.5418 \AA)$ radiation in Bragg-Brentano geometry.

Theoretical Model. Calculations were performed by density functional theory as implemented in the VASP code. ${ }^{57-60}$ We employed the Perdew-Burke-Ernzerhof $(\mathrm{PBE})^{61}$ flavor of the GGA exchange correlation functional along with the projector-augmented wave (PAW) potentials. ${ }^{62,63}$ Considering the layered nature of the systems investigated here, we included the zero damping DFT-D3 (Becke-Johnson) method of Grimme to take into account the longrange interactions. ${ }^{64,65}$ Residual forces after optimization were below $0.01 \mathrm{eV} / \AA$. Two supercells were considered as replica of the pristine monolayer (ML) obtained from the bulk optimization of both $\mathrm{GeS}$ and $\mathrm{GeS}_{2}$. In particular, a $1 \times 4 \times 4$ supercell has been considered for $\operatorname{GeS}(b=14.71 \AA ; c=17.42 \AA)$ and a $2 \times 1 \times 1$ supercell $(a=13.45$ $\AA ; \quad b=16.13 \AA$ ) for $\mathrm{GeS}_{2}$ with a sufficiently thick vacuum $(>20 \AA)$ added to avoid spurious interactions among replicas along the nonperiodic direction ( $a$ for $\mathrm{GeS}, c$ for $\mathrm{GeS}_{2}$ ). Moving from the ML supercell, a quadrilayer (4L) has been assembled and optimized to investigate the processes involved in the oxidation of the two systems. Concerning the sampling of the Brillouin zone, we have adopted a $1 \times$ $6 \times 4$ and a $6 \times 4 \times 1 \Gamma$-centered mesh for the supercells of GeS and $\mathrm{GeS}_{2}$, respectively, samplings that ensure an optimal convergence.

\section{ASSOCIATED CONTENT}

\section{Supporting Information}

The Supporting Information is available free of charge at https://pubs.acs.org/doi/10.1021/acsnano.0c00836.

Optical images of a GeAs upon thinning using the oxidation/etching method and the analysis; AFM images of the substrate; thickness evolution of GeAs with different oxidation/etching time; AFM characterization of GeAs flakes upon incomplete oxidation of the top layer; Raman spectra of $\mathrm{GeS}_{2}$ and $\mathrm{GeS}$ before and after oxidation/etching; thickness evolution of $\mathrm{GeS}$ upon oxidation/etching thinning with shorter oxidation time; AFM images of GeAs showing corrosion of the surface after immersion in DI water without $\mathrm{N}_{2}$ bubbling; details of the theoretical calculations of the oxidation tendency of $\mathrm{GeS}_{2}$ and $\mathrm{GeS}$; discussion on the formation of substoichiometric oxides, and interfacial strain upon oxidation (PDF)

\section{AUTHOR INFORMATION}

\section{Corresponding Author}

Luca Camilli - Department of Physics, Technical University of Denmark, 2800 Kgs. Lyngby, Denmark; Dipartimento di Fisica, Università degli studi di Roma "Tor Vergata", 00133 Roma, Italy; ○ orcid.org/0000-0003-2498-0210; Email: 1cam@ dtu.dk 


\section{Authors}

Jianbo Sun - Department of Physics, Technical University of Denmark, 2800 Kgs. Lyngby, Denmark; 이이이.org/00000001-9741-8726

Giacomo Giorgi - Dipartimento di Ingegneria Civile ed Ambientale, Università degli Studi di Perugia, 06125 Perugia, Italy; Istituto di Scienze e Tecnologie Chimiche "Giulio Natta", Consiglio Nazionale delle Ricerche, 06123 Perugia, Italy; ๑ orcid.org/0000-0003-4892-7908

Maurizia Palummo - Dipartimento di Fisica, Università degli studi di Roma "Tor Vergata", 00133 Roma, Italy; Istituto Nazionale di Fisica Nucleare, 00133 Roma, Italy; (1) orcid.org/0000-0002-3097-8523

Peter Sutter - Department of Electrical and Computer Engineering, University of Nebraska-Lincoln, Lincoln, Nebraska 68588, United States; 10 orcid.org/0000-0002-3301-309X

Maurizio Passacantando - Department of Physical and Chemical Science, University of L'Aquila, 67100 L'Aquila, Italy; SuPerconducting and Other INnovative Materials and Devices Institute (SPIN), Department of Physical Sciences and Technologies of Matter, Consiglio Nazionale delle Ricerche, 67100 L'Aquila, Italy; (1) orcid.org/0000-0002-3680-5295

Complete contact information is available at:

https://pubs.acs.org/10.1021/acsnano.0c00836

\section{Notes}

The authors declare no competing financial interest.

\section{ACKNOWLEDGMENTS}

This research is supported by the Villum Fonden through the Young Investigator Program (Project No. 19130). L.C. acknowledges support from the Italian Ministry of Education, University and Research (MIUR) via "Programma per Giovani Ricercatori - Rita Levi Montalcini 2017”. G.G. and M.P. acknowledge PRACE, ISCRA--B and -C initiatives for awarding access to computing resources on Marconi at CINECA, Italy. P.S. was supported by the U.S. Department of Energy, Office of Science, Basic Energy Sciences, under Award No. DE-SC0016343.

\section{REFERENCES}

(1) Novoselov, K. S.; Geim, A. K.; Morozov, S. V.; Jiang, D.; Zhang, Y.; Dubonos, S. V.; Grigorieva, I. V.; Firsov, A. A. Electric Field Effect in Atomically Thin Carbon Films. Science 2004, 306, 666-669.

(2) Novoselov, K. S.; Jiang, D.; Schedin, F.; Booth, T. J.; Khotkevich, V. V.; Morozov, S. V.; Geim, A. K. Two-Dimensional Atomic Crystals. Proc. Natl. Acad. Sci. U. S. A. 2005, 102, 10451-10453.

(3) Li, X. L.; Han, W. P.; Wu, J. B.; Qiao, X. F.; Zhang, J.; Tan, P. H. Layer-Number Dependent Optical Properties of 2D Materials and Their Application for Thickness Determination. Adv. Funct. Mater. 2017, 27, 1604468.

(4) Sangwan, V. K.; Hersam, M. C. Electronic Transport in TwoDimensional Materials. Annu. Rev. Phys. Chem. 2018, 69, 299-325.

(5) Butler, S. Z.; Hollen, S. M.; Cao, L.; Cui, Y.; Gupta, J. A.; Gutié rrez, H. R.; Heinz, T. F.; Sae Hong, S.; Huang, J.; Ismach, A. F.; Johnston-Halperin, E.; Kuno, M.; Plashnitsa, V. V.; Robinson, R. D.; Ruoff, R. S.; Salahuddin, S.; Shan, J.; Shi, L.; Michael Spencer, O. G.; Torrones, M.; et al. Progress, Challenges, and Opportunities in TwoDimensional Materials beyond Graphene. ACS Nano 2013, 7, 28982926.

(6) Jeong, M. H.; Kwak, D. H.; Ra, H. S.; Lee, A. Y.; Lee, J. S. Realizing Long-Term Stability and Thickness Control of Black Phosphorus by Ambient Thermal Treatment. ACS Appl. Mater. Interfaces 2018, 10, 19069-19075.
(7) Lee, H. S.; Min, S. W.; Chang, Y. G.; Park, M. K.; Nam, T.; Kim, H.; Kim, J. H.; Ryu, S.; Im, S. $\mathrm{MoS}_{2}$ Nanosheet Phototransistors with Thickness-Modulated Optical Energy Gap. Nano Lett. 2012, 12, 3695-3700.

(8) Jia, J.; Jang, S. K.; Lai, S.; Xu, J.; Choi, Y. J.; Park, J. H.; Lee, S. Plasma-Treated Thickness-Controlled Two-Dimensional Black Phosphorus and Its Electronic Transport Properties. ACS Nano 2015, 9, 8729-8736.

(9) Liu, Y.; Nan, H.; Wu, X.; Pan, W.; Wang, W.; Bai, J.; Zhao, W.; Sun, L.; Wang, X.; Ni, Z. Layer-by-Layer Thinning of $\mathrm{MoS}_{2}$ by Plasma. ACS Nano 2013, 7, 4202-4209.

(10) Wang, D.; Wang, Y.; Chen, X.; Zhu, Y.; Zhan, K.; Cheng, H.; Wang, X. Layer-by-Layer Thinning of Two-Dimensional $\mathrm{MoS}_{2}$ Films by Using a Focused Ion Beam. Nanoscale 2016, 8, 4107-4112.

(11) Hu, L.; Shan, X.; Wu, Y.; Zhao, J.; Lu, X. Laser Thinning and Patterning of $\mathrm{MoS}_{2}$ with Layer-by-Layer Precision. Sci. Rep. 2017, 7, 15538.

(12) Huang, Y.; Qiao, J.; He, K.; Bliznakov, S.; Sutter, E.; Chen, X.; Luo, D.; Meng, F.; Su, D.; Decker, J.; Ji, W.; Ruoff, R. S.; Sutter, P. Interaction of Black Phosphorus with Oxygen and Water. Chem. Mater. 2016, 28, 8330-8339.

(13) Zhao, P.; Wang, R.; Lien, D.; Zhao, Y.; Kim, H.; Cho, J.; Ahn, G. H.; Javey, A. Scanning Probe Lithography Patterning of Monolayer Semiconductors and Application in Quantifying Edge Recombination. Adv. Mater. 2019, 31, 1900136.

(14) Zhao, H.; Mao, Y.; Mao, X.; Shi, X.; Xu, C.; Wang, C.; Zhang, S.; Zhou, D. Band Structure and Photoelectric Characterization of GeSe Monolayers. Adv. Funct. Mater. 2018, 28, 1704855.

(15) Jung, C. S.; Kim, D.; Cha, S.; Myung, Y.; Shojaei, F.; Abbas, H. G.; Lee, J. A.; Cha, E. H.; Park, J.; Kang, H. S. Two-Dimensional GeAs with a Visible Range Band Gap. J. Mater. Chem. A 2018, 6, 9089-9098.

(16) Yang, S.; Yang, Y.; Wu, M.; Hu, C.; Shen, W.; Gong, Y.; Huang, L.; Jiang, C.; Zhang, Y.; Ajayan, P. M. Highly In-Plane Optical and Electrical Anisotropy of 2D Germanium Arsenide. Adv. Funct. Mater. 2018, 28, 1707379.

(17) Guo, J.; Liu, Y.; Ma, Y.; Zhu, E.; Lee, S.; Lu, Z.; Zhao, Z.; Xu, C.; Lee, S. J.; Wu, H.; Kovnir, K.; Huang, Y.; Duan, X. Few-Layer GeAs Field-Effect Transistors and Infrared Photodetectors. Adv. Mater. 2018, 30, 1705934.

(18) Abate, Y.; Akinwande, D.; Gamage, S.; Wang, H.; Snure, M.; Poudel, N.; Cronin, S. B. Recent Progress on Stability and Passivation of Black Phosphorus. Adv. Mater. 2018, 30, 1704749.

(19) Cai, Y.; Chuu, C. P.; Wei, C. M.; Chou, M. Y. Stability and Electronic Properties of Two-Dimensional Silicene and Germanene on Graphene. Phys. Rev. B: Condens. Matter Mater. Phys. 2013, 88, 245408.

(20) Li, L.; Gong, P.; Sheng, D.; Wang, S.; Wang, W.; Zhu, X.; Shi, X.; Wang, F.; Han, W.; Yang, S.; Liu, K.; Li, H.; Zhai, T. Highly InPlane Anisotropic 2D $\mathrm{GeAs}_{2}$ for Polarization-Sensitive Photodetection. Adv. Mater. 2018, 30, 1804541.

(21) Prabhakaran, K.; Ogino, T. Oxidation of Ge(100) and Ge(111) Surfaces: An UPS and XPS Study. Surf. Sci. 1995, 325, 263-271.

(22) Tabet, N.; Faiz, M.; Hamdan, N. M.; Hussain, Z. High Resolution XPS Study of Oxide Layers Grown on Ge Substrates. Surf. Sci. 2003, 523, 68-72.

(23) de los Santos Valladares, L.; Dominguez, A. B.; Ionescu, A.; Brown, A.; Sepe, A.; Steiner, U.; Quispe, O. A.; Holmes, S.; Majima, Y.; Langford, R.; Barnes, C. H. W. Thermal Oxidation of Amorphous Germanium Thin Films on $\mathrm{SiO}_{2}$ Substrates. Semicond. Sci. Technol. 2016, 31, 125017.

(24) Moulder, J. F.; Stickle, W. F.; Sobol, P. E.; Bomben, K. D. Handbook of X-Ray Photoelectron Spectroscopy: A Reference Book of Standard Spectra for Identification and Interpretation of XPS Data; Chastain, J., Ed.; PerkinElmer: Eden Prairie, MN, 1992; pp 94-95.

(25) Onsia, B.; Caymax, M.; Conard, T.; De Gendt, S.; De Smedt, F.; Delabie, A.; Gottschalk, C.; Heyns, M. M.; Green, M.; Lin, S.; Mertens, P. W.; Tsai, W.; Vinckier, C. A Study of the Influence of 
Typical Wet Chemical Treatments on the Germanium Wafer Surface. Solid State Phenom. 2005, 103-104, 19-22.

(26) Grossi, V.; Ottaviano, L.; Santucci, S.; Passacantando, M. XPS and SEM Studies of Oxide Reduction of Germanium Nanowires. J. Non-Cryst. Solids 2010, 356, 1988-1993.

(27) Almuslem, A. S.; Hanna, A. N.; Yapici, T.; Wehbe, N.; Diallo, E. M.; Kutbee, A. T.; Bahabry, R. R.; Hussain, M. M. Water Soluble Nano-Scale Transient Material Germanium Oxide for Zero Toxic Waste Based Environmentally Benign Nano-Manufacturing. Appl. Phys. Lett. 2017, 110, No. 074103.

(28) Pokrovski, G. S.; Schott, J. Thermodynamic Properties of Aqueous $\mathrm{Ge}(\mathrm{IV})$ Hydroxide Complexes from 25 to $350^{\circ} \mathrm{C}$ : Implications for the Behavior of Germanium and the $\mathrm{Ge} / \mathrm{Si}$ Ratio in Hydrothermal Fluids. Geochim. Cosmochim. Acta 1998, 62, 16311642.

(29) Li, F.; Liu, X.; Wang, Y.; Li, Y. Germanium Monosulfide Monolayer: A Novel Two-Dimensional Semiconductor with a High Carrier Mobility. J. Mater. Chem. C 2016, 4, 2155-2159.

(30) Tan, D.; Lim, H. E.; Wang, F.; Mohamed, N. B.; Mouri, S.; Zhang, W.; Miyauchi, Y.; Ohfuchi, M.; Matsuda, K. Anisotropic Optical and Electronic Properties of Two-Dimensional Layered Germanium Sulfide. Nano Res. 2017, 10, 546-555.

(31) Yang, Y.; Liu, S. C.; Yang, W.; Li, Z.; Wang, Y.; Wang, X.; Zhang, S.; Zhang, Y.; Long, M.; Zhang, G.; Xue, D.-J.; Hu, J. S.; Wan, L. J. Air-Stable In-Plane Anisotropic $\mathrm{GeSe}_{2}$ for Highly PolarizationSensitive Photodetection in Short Wave Region. J. Am. Chem. Soc. 2018, 140, 4150-4156.

(32) Sutter, E.; Zhang, B.; Sun, M.; Sutter, P. Few-Layer to Multilayer Germanium(II) Sulfide: Synthesis, Structure, Stability, and Optoelectronics. ACS Nano 2019, 13, 9352-9362.

(33) Gomes, L. C.; Carvalho, A.; Castro Neto, A. H. Vacancies and Oxidation of Two-Dimensional Group-IV Monochalcogenides. Phys. Rev. B: Condens. Matter Mater. Phys. 2016, 94, No. 054103.

(34) Barja, S.; Refaely-Abramson, S.; Schuler, B.; Qiu, D. Y.; Pulkin, A.; Wickenburg, S.; Ryu, H.; Ugeda, M. M.; Kastl, C.; Chen, C.; Hwang, C.; Schwartzberg, A.; Aloni, S.; Mo, S. K.; Frank Ogletree, D.; Crommie, M. F.; Yazyev, O. V.; Louie, S. G.; Neaton, J. B.; WeberBargioni, A. Identifying Substitutional Oxygen as a Prolific Point Defect in Monolayer Transition Metal Dichalcogenides. Nat. Commun. 2019, 10, 3382.

(35) Komsa, H. P.; Kotakoski, J.; Kurasch, S.; Lehtinen, O.; Kaiser, U.; Krasheninnikov, A. V. Two-Dimensional Transition Metal Dichalcogenides under Electron Irradiation: Defect Production and Doping. Phys. Rev. Lett. 2012, 109, No. 035503.

(36) KC, S.; Longo, R. C.; Wallace, R. M.; Cho, K. Surface Oxidation Energetics and Kinetics on $\mathrm{MoS}_{2}$ Monolayer. J. Appl. Phys. 2015, 117, 135301.

(37) Lu, J.; Carvalho, A.; Chan, X. K.; Liu, H.; Liu, B.; Tok, E. S.; Loh, K. P.; Castro Neto, A. H.; Sow, C. H. Atomic Healing of Defects in Transition Metal Dichalcogenides. Nano Lett. 2015, 15, 35243532.

(38) Binder, J. F.; Broqvist, P.; Pasquarello, A. Electron Trapping in Substoichiometric Germanium Oxide. Appl. Phys. Lett. 2010, 97, No. 092903.

(39) La Magna, A.; Nicotra, G.; Bongiorno, C.; Spinella, C.; Grimaldi, M. G.; Rimini, E.; Caristia, L.; Coffa, S. Role of the Internal Strain on the Incomplete $\mathrm{Si} / \mathrm{SiO}_{2}$ Phase Separation in Substoichiometric Silicon Oxide Films. Appl. Phys. Lett. 2007, 90, 183101.

(40) Hamann, D. Energetics of Silicon Suboxides. Phys. Rev. B: Condens. Matter Mater. Phys. 2000, 61, 9899-9901.

(41) Yaacoub, L.; Schamm-Chardon, S.; Ovsyuk, N. N.; Zwick, A.; Groenen, J. Studying Thin Ge Films and $\mathrm{Ge} / \mathrm{GeO}_{2}$ Interfaces by Means of Raman-Brillouin Scattering. Bull. Russ. Acad. Sci.: Phys. 2015, 79, 1397-1401.

(42) Lee, J. S.; Bishop, S. R.; Kaufman-Osborn, T.; Chagarov, E.; Kummel, A. C. Monolayer Passivation of $\mathrm{Ge}(100)$ Surface via Nitridation and Oxidation. ECS Trans. 2010, 33, 447-454.
(43) Liu, M.; Jin, P.; Xu, Z.; Hanaor, D. A. H.; Gan, Y.; Chen, C. Q. Two-Dimensional Modeling of the Self-Limiting Oxidation in Silicon and Tungsten Nanowires. Theor. Appl. Mech. Lett. 2016, 6, 195-199.

(44) Khalilov, U.; Pourtois, G.; van Duin, A. C. T.; Neyts, E. C. SelfLimiting Oxidation in Small-Diameter Si Nanowires. Chem. Mater. 2012, 24, 2141-2147.

(45) Heidemeyer, H.; Single, C.; Zhou, F.; Prins, F. E.; Kern, D. P.; Plies, E. Self-Limiting and Pattern Dependent Oxidation of Silicon Dots Fabricated on Silicon-on-Insulator Material. J. Appl. Phys. 2000, $87,4580-4585$.

(46) Dalla Torre, J.; Bocquet, J. L.; Limoge, Y.; Crocombette, J. P.; Adam, E.; Martin, G.; Baron, T.; Rivallin, P.; Mur, P. Study of SelfLimiting Oxidation of Silicon Nanoclusters by Atomistic Simulations. J. Appl. Phys. 2002, 92, 1084-1094.

(47) Castellanos-Gomez, A.; Vicarelli, L.; Prada, E.; Island, J. O.; Narasimha-Acharya, K. L.; Blanter, S. I.; Groenendijk, D. J.; Buscema, M.; Steele, G. A.; Alvarez, J. V.; Zandbergen, H. W.; Palacios, J. J.; van der Zant, H. S. J. Isolation and Characterization of Few-Layer Black Phosphorus. 2D Mater. 2014, 1, No. 025001.

(48) Doganov, R. A.; O’Farrell, E. C. T.; Koenig, S. P.; Yeo, Y.; Ziletti, A.; Carvalho, A.; Campbell, D. K.; Coker, D. F.; Watanabe, K.; Taniguchi, T.; Neto, A. H. C.; Özyilmaz, B. Transport Properties of Pristine Few-Layer Black Phosphorus by van der Waals Passivation in an Inert Atmosphere. Nat. Commun. 2015, 6, 6647.

(49) Island, J. O.; Steele, G. A.; van der Zant, H. S. J.; CastellanosGomez, A. Environmental Instability of Few-Layer Black Phosphorus. $2 D$ Mater. 2015, 2, No. 011002.

(50) Wild, S.; Lloret, V.; Vega-Mayoral, V.; Vella, D.; Nuin, E.; Siebert, M.; Koleśnik-Gray, M.; Löffler, M.; Mayrhofer, K. J. J.; Gadermaier, C.; Krstić, V.; Hauke, F.; Abellán, G.; Hirsch, A. Monolayer Black Phosphorus by Sequential Wet-Chemical Surface Oxidation. RSC Adv. 2019, 9, 3570-3576.

(51) Zhao, Q.; Frisenda, R.; Gant, P.; Perez de Lara, D.; Munuera, C.; Garcia-Hernandez, M.; Niu, Y.; Wang, T.; Jie, W.; CastellanosGomez, A. Toward Air Stability of Thin GaSe Devices: Avoiding Environmental and Laser-Induced Degradation by Encapsulation. Adv. Funct. Mater. 2018, 28, 1805304.

(52) Liu, Y.; Tan, C.; Chou, H.; Nayak, A.; Wu, D.; Ghosh, R.; Chang, H. Y.; Hao, Y.; Wang, X.; Kim, J. S.; Piner, R.; Ruoff, R. S.; Akinwande, D.; Lai, K. Thermal Oxidation of $\mathrm{WSe}_{2}$ Nanosheets Adhered on $\mathrm{SiO}_{2} / \mathrm{Si}$ Substrates. Nano Lett. 2015, 15, 4979-4984.

(53) Yamamoto, M.; Dutta, S.; Aikawa, S.; Nakaharai, S.; Wakabayashi, K.; Fuhrer, M. S.; Ueno, K.; Tsukagoshi, K. SelfLimiting Layer-by-Layer Oxidation of Atomically Thin $\mathrm{WSe}_{2}$. Nano Lett. 2015, 15, 2067-2073.

(54) De Sanctis, A.; Amit, I.; Hepplestone, S. P.; Craciun, M. F.; Russo, S. Strain-Engineered Inverse Charge-Funnelling in Layered Semiconductors. Nat. Commun. 2018, 9, 1652.

(55) Dago, A. I.; Ryu, Y. K.; Garcia, R. Sub-20 nm Patterning of Thin Layer $\mathrm{WSe}_{2}$ by Scanning Probe Lithography. Appl. Phys. Lett. 2016, 109, 163103.

(56) Lu, J.; Wu, J.; Carvalho, A.; Ziletti, A.; Liu, H.; Tan, J.; Chen, Y.; Castro Neto, A. H.; Özyilmaz, B.; Sow, C. H. Bandgap Engineering of Phosphorene by Laser Oxidation toward Functional 2D Materials. ACS Nano 2015, 9, 10411-10421.

(57) Kresse, G.; Hafner, J. Ab Initio Molecular Dynamics for OpenShell Transition Metals. Phys. Rev. B: Condens. Matter Mater. Phys. 1993, 48, 13115-13118.

(58) Kresse, G.; Hafner, J. Ab Initio Molecular-Dynamics Simulation of the Liquid-Metal-Amorphous-Semiconductor Transition in Germanium. Phys. Rev. B: Condens. Matter Mater. Phys. 1994, 49, $14251-14269$.

(59) Kresse, G.; Furthmüller, J. Efficiency of Ab-Initio Total Energy Calculations for Metals and Semiconductors Using a Plane-Wave Basis Set. Comput. Mater. Sci. 1996, 6, 15-50.

(60) Kresse, G.; Furthmüller, J. Efficient Iterative Schemes for $A b$ Initio Total-Energy Calculations Using a Plane-Wave Basis Set. Phys. Rev. B: Condens. Matter Mater. Phys. 1996, 54, 11169-11186. 
(61) Perdew, J. P.; Burke, K.; Ernzerhof, M. Generalized Gradient Approximation Made Simple. Phys. Rev. Lett. 1996, 77, 3865-3868. (62) Blöchl, P. E. Projector Augmented-Wave Method. Phys. Rev. B: Condens. Matter Mater. Phys. 1994, 50, 17953-17979.

(63) Kresse, G.; Joubert, D. From Ultrasoft Pseudopotentials to the Projector Augmented-Wave Method. Phys. Rev. B: Condens. Matter Mater. Phys. 1999, 59, 1758-1775.

(64) Grimme, S.; Antony, J.; Ehrlich, S.; Krieg, H. A Consistent and Accurate $A b$ Initio Parametrization of Density Functional Dispersion Correction (DFT-D) for the 94 Elements H-Pu. J. Chem. Phys. 2010, 132, 154104.

(65) Grimme, S.; Ehrlich, S.; Goerigk, L. Effect of the Damping Function in Dispersion Corrected Density Functional Theory. J. Comput. Chem. 2011, 32, 1456-1465. 